\title{
Performance Analysis of a Mobility Support Scheme between Subnetworks in Differentiated Services*
}

\author{
Jongoh $\mathrm{Kim}^{1}$, Jonghyun $\mathrm{Choi}^{2}$, and Youngsong Mun ${ }^{2}$ \\ ${ }^{1}$ Samsung Electronics Co. Ltd., \\ Suwon Si, Gyeongi Do, Korea, \\ Fido@samsung.com \\ ${ }^{2}$ School of Computer Science, Soongsil University, \\ Sangdo 5 Dong, Dongjak Gu, Seoul, Korea \\ wide@sunny.ssu.ac.kr, mun@computing.ssu.ac.kr
}

\begin{abstract}
The Differentiated Services architecture does not have any provision to support the mobile host. This paper analyzes the performance of a scheme to support the mobility service in the Diff-Serv using the Mobile IP. As results, it is shown that the cost varies around 2 times of that of the Mobile IP. Premium service shows higher costs than Assured service does. It is also shown that packet drop probability for mobile hosts may be managed negligible if the proposed scheme is used.
\end{abstract}

\section{Introduction}

Internet Service Provider (ISP) traditionally has provided the same level of service to all customers, namely the best-effort service. The increased internet usage resulted in a performance hit of highly mission critical applications. At the same time, newer applications that need a better service quality have emerged.

The Differentiated Services (Diff-Serv) [1] is an effective approach for Quality of Service (QoS) support in the internet. The Diff-Serv is expected to provide a better QoS control with scalability than existing schemes today. However, the Diff-Serv in its current form does not have any provision for the mobility services.

Recent advances in the wireless network interfaces and the implementation of the global networks make host mobility an issue of interest both in wireless and wired networks. Thus, in this paper a scheme to support the mobility service in the Diff-Serv is proposed.

The paper is organized as follows. In section 2, related works are briefly overviewed. Proposed mobility scheme in the Diff-Serv architecture is described in Section 3. The network architecture is described and operations for supporting the mobility service in the Diff-Serv using the Mobile IP [2] are proposed. In section 4, the

* This work was supported by Korea Research Foundation Grant (KRF-2000-041-E00266)

Dr. Mun is the corresponding author. 
performance analysis is performed. Results of the performance analysis are given in section 5. Finally, the conclusion is given in section 6 .

\section{Related Works}

The Mobile IP is proposed to enable nodes to change their point of attachment to the internet without changing their IP address [2]. R. Jain et al. performed the performance analysis of the Mobile IP [3]. However, they did not consider the cost incurred by the packets from the mobile host $(\mathrm{MH})$ to the correspondent host $(\mathrm{CH})$.

S. Black et al. proposed Diff-Serv [1]. Diff-Serv defines the layout of the TOS byte (DS field) [4] and a base set of packet forwarding treatments (per-hop behaviors, or PHBs) [5]. By marking the DS fields of packets differently and handling packets based on their DS fields, several differentiated services becomes essentially a relativepriority scheme.

In order for a customer to receive the differentiated services from its ISP, he/she must have a service level agreement (SLA) with the ISP. The Premium service and its corresponding Expedited Forwarding PHB provides a guaranteed bandwidth, low delay and low loss service that show the same characteristics as a virtual leased line [6]. The Assured service proposed in [7] only permits a statistically guaranteed rate. It permits to use additional available capacity while providing a base rate. Packets that exceed the negotiated rate are either marked as the best-effort traffic or dropped. The overall dropping probability of Assured service packets is considerably lower than for best-effort packets.

M. May proposed a simple model for the performance analysis of Diff-Serv [8]. Each service class using queue models was analyzed.

\section{Proposed Scheme}

The network architecture premised in this paper is the same one defined in [9] with the bandwidth broker (BB) [10]. In a domain, there may be more than two subnetworks. For the mobility support there should be agents defined in the Mobile IP in each subnetwork. The leaf router marks packets. The BB located in a domain is responsible for allocating the preferred service to users as requested, and for configuring the network routers with the correct forwarding behavior for the defined service. A BB is associated with a particular trust region, one per domain. Only BB can configure the leaf routers to deliver a particular service to flows. The configuration request might come from another region's BB. The BB is used for allocation of the service profile of $\mathrm{MH}$.

The range of the movement of $\mathrm{MH}$ in the Diff-Serv may be categorized into four groups such as the movements between subnetworks in a same domain, between customer domain, between provider domains, and returning to home domain. When $\mathrm{MH}$ moves from one subnetwork to another, the leaf router which $\mathrm{MH}$ will be connected to is not yet configured according to its service profile. Thus the QoS is not guaranteed. 
The following describes the registration procedure for the movement between subnetworks as shown in Fig. 1 [11].

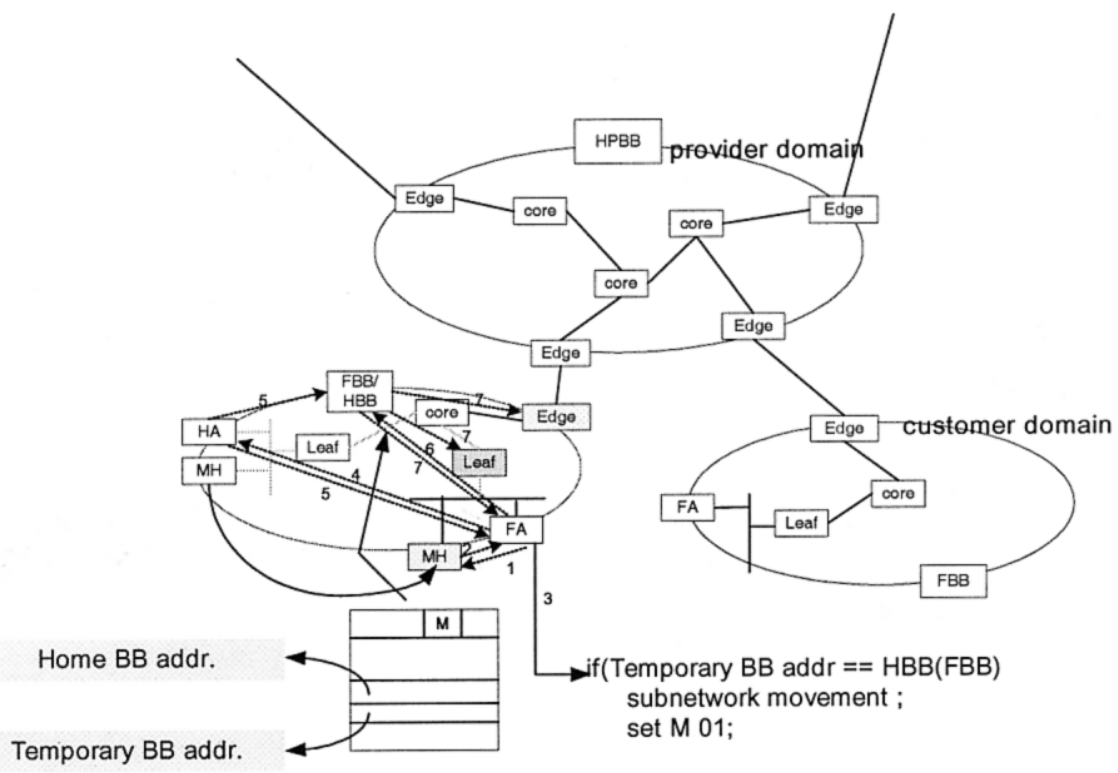

Fig. 1. Registration procedure for the movement of $\mathrm{MH}$

(1) After $\mathrm{MH}$ moves, it detects its movement by the received advertisement messages.

(2) $\mathrm{MH}$ forwards a registration message to a foreign agent (FA). At this time, $\mathrm{MH}$ uses a modified registration request message having the address fields of the home $\mathrm{BB}$ and the temporary BB. It is assumed that the address of the home BB is already known to $\mathrm{MH}$ and the first temporary $\mathrm{BB}$ address is set as the home $\mathrm{BB}$ address. To obtain the temporary $\mathrm{BB}$ address for $\mathrm{MH}$, the $\mathrm{FA}$ receiving the registration reply message writes the address of its domain's BB in the message and forwards it to $\mathrm{MH}$. MH records this address and do not update it until receiving another registration reply message which contains a different temporary BB address.

(3) If the BB address which is known to the FA is the same as the temporary BB address, a FA determines that MH has moved between subnetworks.

(4) If the FA determines that $\mathrm{MH}$ has moved between subnetworks, it forwards the registration request message with which the flag $M$ is set to value 01 to the HA. The 2 bits of the code field in the registration request/reply message are used for flag $M$. If the flag $M$ is not supported in the Diff-Serv, MH forwards the registration request message, where home and temporary BB address fields are filled up zeros, to the FA. Then, the FA compares these two addresses. If they are all zeros, flag $M$ is set to 00 and then the general Mobile IP operations are triggered. If it is 10 , the movement between (customer/provider) domains is assumed. In case of the movement between subnetworks, MH's profile is already enrolled in the BB. Thus, it is not necessary to acquire MH's profile. 
(5) After the home agent (HA) sent the registration reply message to the FA, to keep $\mathrm{CH}$ 's service in tunneled path, following procedure is needed. The HA sends the service request to the home $\mathrm{BB}$ for the path reconfiguration in the tunneled route. Then, the home $\mathrm{BB}$ relays the service request to the home provider $\mathrm{BB}$. If the service request reaches to the $\mathrm{BB}$ that is located in a domain where the FA is, the BB makes an admission control decision and comes to configure the classification and policing rules on the leaf and boarder routers. If the request is denied, an error message is sent back to the provider BB. After this procedure is done successfully, the foreign provider $\mathrm{BB}$ will configure the classification, policing and reshaping rules on the egress and ingress routers. Then, the home BB and home provider BBs are also set the policing and reshaping rule on the leaf and boarder router.

(6) If the FA receives the registration reply message to grant the request, it sends the service request message to the foreign $\mathrm{BB}$ in place of $\mathrm{MH}$. Then the foreign $\mathrm{BB}$ and foreign provider BBs will configure the leaf and boarder routers.

(7) When the FA receives the reply to grant the service request from the foreign $\mathrm{BB}$, it sends the registration reply message with an address of temporary BB to $\mathrm{MH}$.

\section{Performance Analysis}

The distances between the various entities involved in the proposed scheme are shown in Fig. 2. This system model is for the performance analysis in the movement between subnetworks.

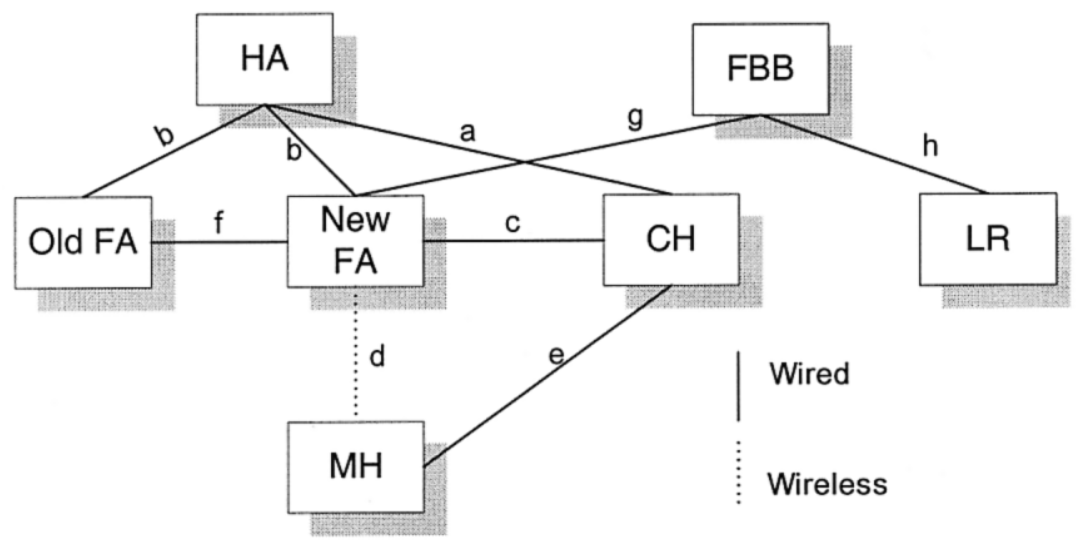

Fig. 2. System model of the proposed scheme

For the proposed scheme, during the time interval when the $\mathrm{MH}$ moves between the subnetworks in a domain, the total cost incurred, $C_{S M D S}$ is given by (1).

$$
C_{S M D S}=C_{r g}+C_{o l d F A}+C_{n e w F A}+C_{p k t}
$$


The cost of $\mathrm{MH}$ registering at the new subnetwork, $C_{r g}$ is given by (2). Besides the cost of $\mathrm{MH}$ registering in the Mobile IP, the distances and processing cost that takes place in reconfiguring the leaf router must be added.

$$
C_{r g}=2(b+d)+2(g+h)+5 r+4 r
$$

The cost of data packets lost by being delivered to the old FA during the registering delay is given by (3).

$$
C_{\text {oldFA }}=\alpha_{1} \times t_{r g_{-} S M D S} \times C_{d l}
$$

$C_{d l}$ in the Mobile IP is $l(a+b+d)+2 r$ which means the cost of a single data packet delivered from $\mathrm{CH}$ to $\mathrm{MH}$ via tunneling at the HA [3]. However, in the proposed scheme, the overhead of traffic conditioning in the boarder router $r_{c}$ should be added as given in (4). Here, $n$ and $r_{c}$ mean the number of boarder routers between $\mathrm{CH}$ and $\mathrm{MH}$ and the average processing cost for traffic conditioning in the boarder router, respectively.

$$
C_{d l}=l(a+b+d)+2 r+n r_{c}
$$

For the registration delay, the maximum time among two delivery times $\left(t_{b}, t_{g}+t_{h}\right)$ must be added because the delivery of registration message to a HA coincides with the delivery of service request.

$$
t_{r g_{-} S M D S}=t_{d}+\max \left(t_{b}, t_{g}+t_{h}\right)+5 t_{r}+2 t_{r}
$$

The cost of data packets delivered to the MH via a new FA is the same as defined in the Mobile IP. Thus, $C_{\text {newF }}$ becomes same as in the Mobile IP.

The cost of data packets delivered from $\mathrm{MH}$ to $\mathrm{CH}, C_{p k t}$ is given as follows.

$$
C_{p k t}=\rho_{2} \times e \times D_{\text {Diff-Serv }}
$$

The expected delay, $D_{\text {Diff-Serv }}$ and the drop probability in each hop are dependent on the service class, namely Assured and Premium service. In the next, these two parameters are obtained according to the service class.

\subsection{Expected Delay and Drop Probability in Assured Service}

We assume Random Early Detection with In/Out (RIO) as the buffer management scheme. Assume that the packets arrive in the queue according to a Poisson process with rate $\lambda$. The packets are In packets with probability $p$. The packets require a service exponentially distributed with the parameter $\mu$. The RIO accepts all packets until the queue size is equal to $K / 2$ and drops a packet with the probability that increases linearly to $10 \%$ for tagged packets, and to $90 \%$ for non-tagged packets.

$$
\alpha^{T}(n)=\left\{\begin{array}{cc}
1 & n \leq \frac{2}{K} \\
1-\frac{0.1(2 n-K)}{K} & \frac{2}{K} \leq n \leq K-1
\end{array}\right.
$$

We can also derive $\pi(n)$ using the stationary distribution of buffer contents. 


$$
\pi(n)=\pi(0)\left(\frac{\lambda}{\mu}\right)^{n} \prod_{i=0}^{n-1} \alpha(i)
$$

Let $\pi_{d r o p}^{T}$ be the drop probabilities for tagged packets. The drop probability means that the queue has no room for the packets. The probability of rejecting a packet is equal to 1 minus the probability of accepting it in the queue.

$$
\pi_{d r o p}^{T}=1-\sum_{n=0}^{K} \alpha^{T}(n) \pi(n)
$$

The expected delay in the queue for Assured service is given by (10).

$$
D_{\text {Assured }}=\frac{1}{\mu} \sum_{n=0}^{K-1}(1+n) \pi(n) \alpha(n)
$$

\subsection{Expect Delay and Drop Probability in Premium Service}

The Premium Service can be modeled as follows. The router includes two separate queues, one with the finite size $K$ accessible only to tagged packets. If the tagged queue is full, arriving tagged packets are discarded.

We assume that the input stream is a Poisson with rate $\lambda$, that the arriving packets are tagged with probability $p$. The input stream of the high and low buffers are an independent Poisson process with the rate $\lambda_{1}=\lambda_{p}$. Let $\phi_{1}=\lambda_{1} / \mu$ be the load factor of the tagged queue.

$$
\pi(n)=\pi(0) \phi_{1}^{n} \quad(n \leq K)
$$

For the high priority customers the response time $D_{\text {Premium }}$ is the time of customers in a M/M/1/K queue with arrival rate $\lambda_{1}$. $D_{\text {Premium }}$ is obtained using Little's law [12].

$$
D_{\text {Pr } \text { emium }}=\frac{1}{\mu-\lambda_{1}} \frac{1-(K+1) \phi^{K}+K \phi_{1}^{K+1}}{1-\phi_{1}^{K+1}}
$$

We also obtain the loss probability of the high priority class.

$$
\pi_{1}=\phi_{1}^{K} \frac{\left(1-\phi_{1}\right)}{1-\phi_{1}^{K+1}}
$$

\section{Results}

As results of the performance analysis, $C_{M I P}, C_{S M D S}, C_{N M D S}$, and the drop probability in the leaf router are obtained. Costs of the proposed scheme are shown as normalized to the cost of the Mobile IP. For the roundtrip time calculation in wired and wireless 
links, equations in [3] are used. For parameters, $a=4, b=c=g=h=y=2, d=1$ and $e=6$ are assumed.

The cost ratio, $C_{S M D S}$ to $C_{M I P}$, is shown in Fig. 3. It is showing the cost ratio of the proposed scheme to the Mobile IP in case of the movement between subnetworks. Cost of Assured service is approximately 1.95 times higher that that of the Mobile IP. Cost of Premium service is more than 2 times higher. Since the proposed scheme requires the additional operations to the Mobile IP, it is natural that the cost of the proposed scheme becomes higher than that of the Mobile IP. The cost of Premium service is approximately $10 \%$ higher than that of Assured service as shown in the Fig. 3. As Packet to Mobility Ratio (PMR) [3] is increasing, the cost is decreased. This is because the amount of the data packet relative to the control packet such as the registration and reply packets is increasing as PMR is increasing.

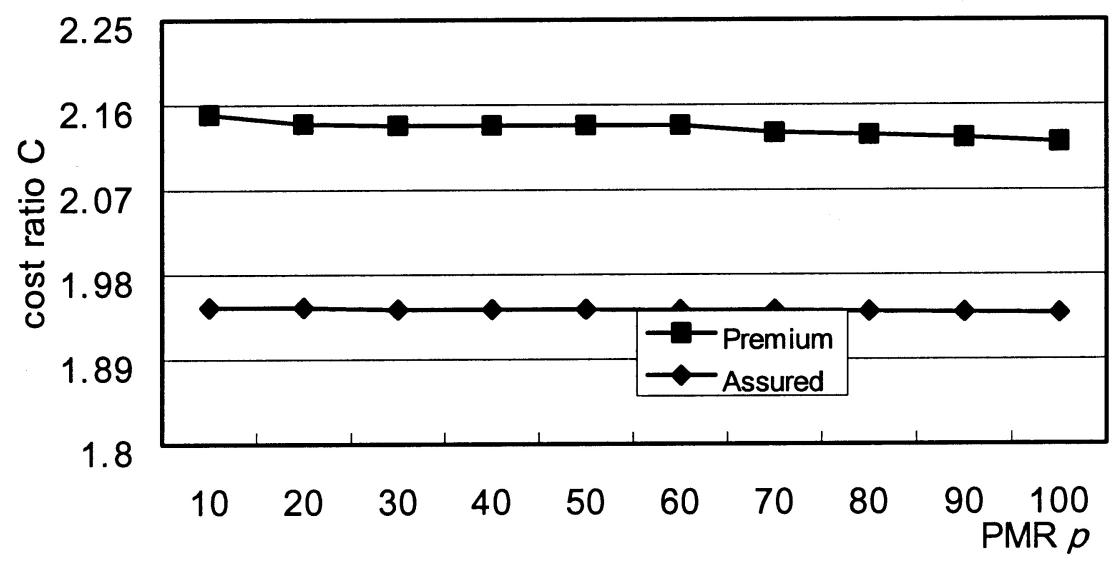

Fig. 3. Cost ratio for the movement of $\mathrm{MH}$

Next, the packet drop probability in the leaf router is shown according to the system load in Fig. 4. The buffer size of 50 and the utilization factor of the queue of 0.5 are assumed. In the plain Diff-Serv architecture, MH's packets will be heavily dropped in the leaf router since the Diff-Serv alone does not support the mobility. However, if the proposed scheme is used, the packet loss may be significantly down. Actually, in Fig. 4 , it is confirmed that there is negligible packet drop probability in the practical system traffic load. 


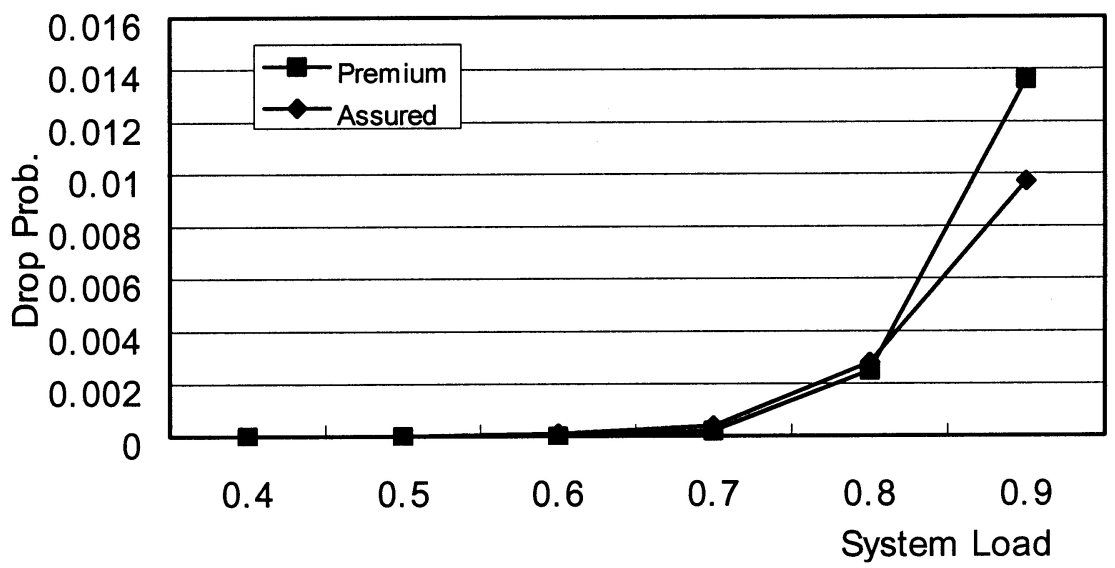

Fig. 4. Packet drop probability

\section{Conclusions}

Over the past several years there has been a considerable amount of research to provide $\mathrm{QoS}$ for the real time and multimedia data service in the internet. Among these researches Differentiated Service model is considered as the practical choice having scalability. However, since this model only considers fixed hosts, mobility service can not be supported.

To support the quality of service and the mobility service in the Internet simultaneously we propose a new scheme, namely the proposed scheme. It is basically a mechanism to support the Mobile IP in the Diff-Serv architecture. Performance analysis is performed to see the cost of the proposed scheme when compared with the cost of the Mobile IP without the Diff-Serv. Through the performance analysis it is shown that the cost varies around 2 times of that of the Mobile IP. Premium service shows higher costs than Assured service does. Packet drop probability shows that there is little packet drop probability for mobile communications in the practical system load if the proposed scheme is used.

\section{References}

1. S. Blake, D. Black, M. Carlson, E. Davies, Z. Wang, and W. Weiss: An Architecture for Differentiated Services, RFC 2475, Dec. 1998.

2. C. Perkins: IP Mobility Support, RFC 2002, Oct. 1996.

3. R. Jain, T. Raleigh, C. Graff and M. Bereschinsky: Mobile Internet Access and QoS Guarantees using Mobile IP and RSVP with Location Registers, in Proc. ICC'98 Conf., pp. 1690-1695, Atlanta.

4. J. Postel: Service Mappings, RFC 795, Sept. 1981. 
5. K. Nichols et al.: Definition of the Differentiated Services Field (DS Field) in the IPv4 and IPv6 Headers, RFC 2474, Dec. 1998.

6. V. Jacobson and K. Poduri: An Expedited Forwarding PHB, RFC2598, June 1999.

7. J. Heinanen, F. Baker and J. Wroclawski: Assured Forwarding PHB Group, RFC 2597, 1999.

8. M. May, J.-C. Bolot, A. Jean-Marie and C. Diot: Simple Performance Models of Differentiated Services Schemes for the Internet, in Proc. INFOCOM'99, pp. 1385-1394, New York.

9. K. Nichols, V. Jacobson and L. Zhang: A Two-Bit Differentiated Services Architecture for the Internet, Internet draft draft-nichols-dif-srv-arch-00.txt, Nov. 1997.

10. X. Xiao and L.M. Ni: Internet QoS: A Big Picture, IEEE Network Magazine, March/April, 1999.

11. Y. Mun and J. Kim: Mobility Support between Subnetworks in Differentiated Services, in Proc. PDPTA 2000, pp. 2079-2085, Las Vegas.

12. L. Kleinrock: Queueing Systems, vol.1. J. Wiley \& Sons, 1975. 